\title{
THE MANAGEMENT OF A PATIENT WITH A COLOSTOMY OR AN ILEOSTOMY
}

\author{
B. N. Brooke, M.Chir., F.R.C.S. \\ The Department of Surgery, Queen Elizabeth Hospital, Birmingham
}

There is one fundamental difference between a left iliac colostomy and an ileostomy: in health solid faeces escape from the former, while only a fluid or, at best, semi-solid motions flow from an ileal stoma. Furthermore, evacuation from an established colostomy is usually under the control of a gastro-colic reflex and occurs only once or twice in a day, whereas it is rare to find an ileostomy entirely subject to this reflex and when it is so evacuation takes place after every meal. More commonly ileal movements continue throughout the day and night at indefinite intervals. There are other differences; the contents of the colon can be detected by their odour but ileal contents do not have this disadvantage except when some element of obstruction arises as a complication. To the patient the control and collection of excreta is of paramount importance. The man with a colostomy may take a fully active part in society with nothing more than a light dressing, provided he can maintain a solid stool and regulate his life so that his bowel actions occur at expected times; but the patient with an ileostomy has no such easy control and for him an apparatus for the collection of faeces is necessary and its adaptation to the ileostomy must remain watertight. To the surgeon, therefore, the actual technical construction of the colostomy is relatively unimportant; provided there is an unimpeded vent all will be well. But an ileostomy, like an amputation stump to which a prosthesis is to be adapted, must be fashioned with the minutest care in order that a bag may be applied to it in a watertight fashion. It requires skill, patience and forethought to ensure that an ileal stoma is efficient; though, for example, it matters little whether a colostomy stoma lies flush with the skin or protrudes, this is just one of the factors which renders an ileostomy unmanageable; though the exact position of a colostomy on the abdominal wall is of small moment, an ileostomy must be sited with care in order that the flange of the adherent bag may not impinge upon such structures as the anterior superior iliac spine, the umbilicus and laparotomy scars. These are only a few of the differences which permit a colostomy to be done almost casually and yet with success, while utter failure will follow ileostomy unless performed in the most meticulous manner.

The management of a patient for colostomy or ileostomy starts before operation. It may seem unnecessary to stress the point that any patient who is to have an artificial anus should be warned of this, but it is surprising how often it is overlooked. It is indefensible on ethical grounds not to allow a patient the opportunity of refusal to undergo such an operation and its outcome. Nevertheless, if acceptance is not obtained the consequences to the patient must be fully and clearly explained: if the patient then persists in his attitude it is probably wise in these days of increasing medicolegal actions to obtain a refusal in writing duly signed. But apart from ethical considerations a preliminary explanation is of value since it engenders a co-operative response facilitating the after-care of the patient. This is of particular importance for ileostomy since its management by the patient is difficult and calls for skill; it will be an irksome task imperfectly performed if the ileostomy life is not accepted in a positive manner. There are two ways of approaching the subject. The patient can be told directly that the surgical cure of his disease, whether it be ulcerative colitis of familial polyposis, will entail the institution of an ileostomy, in all probability permanent. But many patients with ulcerative colitis are young, highly sensitive and are often women, to whom such an idea is abhorrent. To them it is possible to mention, casually at first, that there is an operation for their disease. They may then ask if they can have this operation, for they are ill, incapacitated and well aware that unless something is done they are doomed to inactivity and possibly worse. Alternatively a former patient can be used 
as a decoy, being introduced to the ward as though on a friendly visit to the sister, though in reality being there to reveal that he is restored to health and normal life. The choice of approach must be decided on the type of patient, but whichever method is used it is valuable to arrange before operation an interview between the patient and someone who has a successful ileostomy, in order that doubts and difficulties foreseen can be discussed and dispelled. Even those patients for whom an artificial anus is expected to be only temporary, will be shocked by its revelation on the abdominal wall post-operatively, unless they have received a previous warning.

Certain points in operative technique need to be considered when making a colostbmy since they have a direct bearing on subsequent management. First it must be understood that a colostomy should be placed as far distally in the colon as the lesion will permit, since the further to the right it is situated the looser the motion; it should always lie on the anterior abdominal wall where the patient can manage it easily. The single end, as produced in abdomino-perineal excision of the rectum, is the ideal form of permanent colostomy but if the colon distal to the opening is to be retained then it is preferable to separate the proximal from the distal end with a bridge of skin, for there is sometimes a tendency for faeces to pass into the gut beyond, over the spur of a loop colostomy particularly if this subsides. The para-colic gutter should be closed with a purse-string suture, as recommended by Rankin (1927), since internal herniation and obstruction may otherwise occur at this $s^{\prime} \partial \mathfrak{l}$ though this manoeuvre is not possible with a transverse colostomy. Obstruction at the abdominal wall is avoided by ensuring an adequate incision, which can be assessed by inserting the tips of two fingers, together with relieving incisions placed at right angles to the main axis in such aponeurotic layers as the external oblique. Stenosis, which arises at skin level at a later date, can be avoided by suturing mucosa to skin (Patey, I95 I); should this complication arise it may be dealt with by digital dilatation, but an elliptical excision of the skin will be required if a tight stricture develops. Prolapse and herniation both develop from technical failures, prolapse from leaving the afferent limb too slack in a left iliac colostomy. Whenever a colostomy is implanted in the main incision hernia is prone to follow; this is particularly so when, for example, an abdominoperineal excision is performed through a left iliac 'muscle-cutting' incision. It is therefore better to employ a paramedian approach with a stab incision to accommodate the colostomy. Endeavours have been made to fashion a sphincter by bringing the colostomy out between fibres of the rectus muscle, but all such attempts are fruitless气 since sphincteric mechanisms rely on a sensory arc for their function and the afferent limb of $a$ ำ colostomy can in no way develop that special $\frac{c}{-}$ sense which gives warning of the presence of solid, fluid or gas provided by the terminal two inches of rectum.

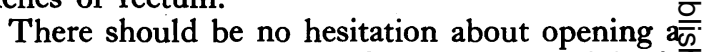
colostomy at the time of operation. This is obligatory in the presence of obstructions; it is safer to do so when there is no pre-operativees obstruction since paralytic ileus is occasionally $\overrightarrow{0}$ induced by the gas distension which occurs during the 48 to 76 hours that the colostomy remainsw closed. (Gabriel and Lloyd-Davies, 1935) Infection of a colostomy wound open from the time of operation is a rarity, more probably due to contamination during the operation than subse--O quent soiling with faeces; in the simple un- $w$ obstructed case a faecal motion seldom passes before the third day, though wind escapes earliercy thus bringing relief to the patient. By the time ao motion occurs the wound has become sealed off by natural processes which are probably effective $\vec{z}$ within twelve hours of operation. There is now need for concern should constipation persist to six or seven days, provided gas is escaping, but $\$ \sigma^{\circ}$ encourage evacuation six to ten ounces of oil magy be introduced through a soft rubber catheter. 등. 응 For a loop colostomy a rod is inserted to supports the spur and is maintained for at least two weeks after that time it can be replaced by a rubber tube according to the method described by Gabrie (1948) if it is necessary to ensure a good persistent spur. If however the colostomy is to be temporary3 the rod is removed on the fifth to seventh post-? operative day. The time has gone when it waso necessary to crush the spur before closing काँ colostomy; this was done as a preliminary to 3 . ' extra-peritoneal ' closure and an enterotome waso employed. This instrument had its dangers there was almost invariably a pyrexial response due to peri-colitis with, sometimes, actual abscesse formation; the colon has been perforated and eveno the ureter has been crushed in the clamp; the resultant scarring in the bowel wall has led tog stricture and obstruction later. Using pre $\frac{\bar{\pi}}{\mathrm{N}}$ operative phthalyl- or succinyl-sulphathiazole, ot streptomycin by mouth, a formal closure can be achieved with perfect safety from within the्ट abdomen and the abdominal wall be repaired; noo antibiotic need be given post-operatively unles there has been much soiling.

It used to be considered necessary for colostomys? patients to wash out the colon daily and this view is still held by some (Benjamin 1954). It is, however, burdensome for the patient, who must copes with douch-cans, catheters and the like each 
morning, and it is not without hazard. Repeated enemata can lead to fatal perforation of the afferent loop (Hoffman and Macht I954), a complication which has been seen as late as 20 years after operation. For all patients, therefore, the aim should be to obtain a natural motion at a regular time each day and it is usual to find that one is passed about half-an-hour after rising in the morning and often another in the evening after a meal. The bowels may be made to move in the morning, if this does not happen naturally, by drinking a cup of tea on waking and smoking a cigarette. Regularity is assured by taking meals at regular intervals and at the same time from day to day. The content of the diet also plays a part and in this respect the patient must experiment for himself; it should be explained to him that there is considerable individual idiosyncrasy in such matters but that many patients find fruit and certain vegetables, such as spinach and onions, are loosening while beer may have the same effect. Once a patient has discovered the diet that suits him best he will find that his bowels act at times he can anticipate and that only the slightest soiling of his dressings occurs in the intervals. The confidence that this gives is considerable and it is to this end that attention should be paid during the latter part of the patient's period in hospital. Colostomy diarrhoea is the greatest incapacity; dietary indiscretions may be a cause and can usually be solved without difficulty. Though it is seldom necessary to do so, a solid motion can also be encouraged with chalk and opium, bismuth, kaolin, charcoal or isogel; amphetamine sulphate, taken after meals so as not to inhibit the appetite, has been suggested (Benjamin 1954). For persistent diarrhoea a further cause must be sought, since it is usually due to an unsuspected condition which can be treated accordingly. A fat balance test may reveal steatorrhoea calling for a reduction in the fat intake, a barium progress meal bring to light an unsuspected Crohn's lesion requiring resection. Previous surgery may play a part; a pre-existing partial gastrectomy can induce a recurrent or persistent diarrheoa very resistant to treatment.

The modern colostomy appliance is not designed to be a receptacle for faeces but a means whereby a dressing may be retained over the opening and support be given to the abdominal wall at this site. This purpose can be achieved with a 'many-tail' bandage and a piece of cardboard incorporated as stiffening in the dressings, but it is convenient to supply a shallow plastic cup as a shield to be held in place by a light narrow belt, or a corset of fabric or elastic. The thick rubber bag with wide mouth, which was at one time provided, was probably necessary for patients who followed the lavage routine in order to collect fluid, some of which was inevitably retained at the time of the enema. It had the disadvantage of weakening the abdominal wall around the stoma, thus leading to hernia and prolapse. All rubber appliances also suffer from the failing that the odour of large bowel contents becomes more offensive on contact with rubber; this is only occasionally encountered with ileostomy discharges which are comparatively odourless. Attempts to use a plastic adherent bag have not met with marked success for the stiff heavy stool from a colostomy tends to cause it to become detached. Indeed such bags are provided on the wrong premise, since the whole management of a colostomy aims at producing a bowel action at certain anticipated times when the patient may evacuate it into a kidney dish or lavatory pan, and at no other occasion.

Whereas the convalescence following colostomy is commonly smooth and subsequent complications are rare, an ileostomy is attended by many dangers, in particular obstruction, and is not infrequently followed by disturbances which render it inefficient (Brooke 1954). The management of the ileostomy must therefore ever be in the mind of the surgeon while performing the operation. As for colostomy, the single end is ideal and this is inevitably achieved in primary colectomy (simultaneous colectomy and ileostomy), but when done as an isolated operation for ulcerative colitis it is wiser to bring out both ileal limbs separately, since obstruction from stricture in the colon may cause a closed distal end to 'blow-out.' A ' loop ' ileostomy, advocated by some (Lahey 195I) in emergency, is indefensible since an adherent bag cannot be applied to it satisfactorily and it is no safer to perform. The prime object at ileostomy is to produce a stoma over which a bag can be placed in satisfactory fashion. It is therefore necessary to place the stoma with care, and again a stab incision is ideal. The adherent bag has a flange, about three inches in diameter, which provides the 'face' for adhesion to the skin; this must not impinge on anterior superior iliac spine, the groin, or distal ileal end, otherwise it will become unseated, and it should it possible avoid any laparotomy scar. The opening may be placed in the right lilac fossa or higher, in which circumstance there must be adequate clearance from the costal margin. Sufficient ileum, about $\mathrm{I} \frac{1}{2}$ inches, should protrude beyond the skin to allow the ileal contents to drop into the bag; if the stoma is short leakage will occur under the flange.

The next object is to avoid complications. There are those, such as obstruction, prolapse and stenosis which can mar a colostomy, but recession or retraction, which has little effect on the efficiency of a colostomy, is a disaster for the ileostomy 
patient since leakage is then inevitable and the patient disabled. Stomal fistula, caused by suturing the wall of ileum to the wound or the chaffing of the flange of the bag which is pushed up against the stoma by flexion of the thigh when the stoma is placed too low, has a similar effect. The skin not infrequently becomes excoriated after operation, so that the bag cannot be applied, and though this soon heals it will not do so if the ileum discharges itself at skin level because of retraction, or through a fistula. The points of technique to be observed are therefore these. All possible sites of internal herniation should be closed, for example the lateral gutter to the left of the exteriorized distal end of the colon in primary colectomy; also the para-ileal gutter and this may be done by a purse-string suture for a double-ended ileostomy, and more simply for the terminal form by sewing the cut edge of mesentery to the parietal peritoneum. The resultant fixation of the mesentery is of importance in preventing recession and prolapse, which can also occur due to hernia when the opening in the abdominal wall is too wide (it is sufficient if the tips of two fingers can be inserted), or the wall itself has become attenuated by infection. If subsequently the ileum prolapses it should under no circumstances be resected; a formal laparotomy is required either to re-attach the mesentery or to replace the stoma to a new site, depending on the cause. The portion of ileum which lies outside the abdominal wall is best everted (Brooke, 1952) and the cut mucosal edge sutured to the skin; the everted end should be about $\mathrm{I}_{2}^{\frac{1}{2}}$ inches in length. This is done not only to reduce the possibility of stenosis but because it provides a supple end which bends over into the bag. If the mesentery below has been adequately attached then no greater tendency to prolapse is induced by this manoeuvre, nor will recession occur.

Immediately following operation attention is directed towards fluid and electrolyte balance. At first the evacuations are very fluid and sudden collapse may occur due to loss of electrolytes. This is countered by saline infusions but it is advisable to anticipate such trouble by giving extra salt before operation and ensuring fluid balance with intravenous saline afterwards. The small bowel acts from 12 to 24 hours after operation and the bag may be glued to the skin in the theatre or applied when the motions start. The skin has to be protected in this manner, or with a dressing, for it is prone to excoriation in these early days. When excoriation does occur the area around the ileostomy may be covered with lint, rather than gauze which is more abrasive, and waterproof adhesive, to which platform the bag is then cemented. Zinc oxide cream, Baltimore Paste or barrier cream have proved useful unguents for use under the lint. Once excoriation has healed it $z$ seldom recurs, provided the stoma remains $\stackrel{\mathbb{Q}}{\varrho}$ efficient, except for a small area of about one- $c$. eighth inch in depth where skin meets mucosa $\vec{\equiv}$ and this can be protected by a ring cut out of lint $\stackrel{?}{\rightarrow}$ and sealed down to the skin by the adherent flange.

After four or five days when a more normal diet 음 is being taken by mouth the ileostomy contents $\frac{5}{\partial}$ begin to thicken, a process which may be en- $\mathbb{\Phi}$ couraged by the administration of 'isogel,' and $๗$ within a short while it is possible for the patient $\vec{\circ}$ to learn to apply the bag himself. It is im-? portant for him to know that the special cement $\vec{\omega}$ should be spread on to both skin and flange not too liberally and be allowed to become tacky before the $\frac{\sigma}{7}$ bag is pressed on-just like mending a puncture. 3 In the first month the lateral side is reinforced with $\omega$ adhesive since leakage occurs at this site on $\omega$ recumbency, more particularly before the patient $\vec{\omega}$ gains weight and the concavity medial to the anterior superior iliac spine fills in.

It is during this period that intestinal obstruction is most likely to occur. It may be due to snaring of a loop of bowel in a gutter, or round an adhesion, 3 or to fixation and kinking of several loops by fine adhesions, as is seen after tuberculous peritonitis, $\vec{\bullet}$ If obstruction is complete, with cessation of wing as well as faeces, laparotomy must be undertake but the majority of these obstructions are if complete and the episodes pass off after 36 to 48 anxious hours. Relief can sometimes be obtained by the passage of a catheter and about $50 \mathrm{ml}$. of $\frac{\circ}{\Phi}$ saline. In any case it is wise to inspect the stoma since this swells with oedema during the first week $\overrightarrow{0}$ and may be the site of obstruction due to strangulation within the opening of the bag.

By the time the patient is ready to leave hospital he must be fully conversant with the $\frac{0}{3}$ intricacies of the bag: at the Queen Elizabeth Hospital, Birmingham, all patients see an instructional film before departure. Two bags are provided, one to be washed and dried while the $\frac{0}{3}$ other is worn, together with tubes of cement and a bottle of its solvent, benzene, for removing the $\frac{\text { ㄱ }}{5}$ bag and cleaning skin and flange. The bag may $\frac{T}{0}$ be worn for as long as three days at a time, for it can be emptied while worn, but many patients o prefer to change it morning and evening for $N$ hygienic reasons. A light elastic or fabric belt 0 lying like a cuff between the flange and the main $\omega$ body of the bag gives extra support by maintaining pressure on the flange. There are two types of adherent bag, those made in one piece and those with flange and detachable body, the latter being ? somewhat more bulky (Counsell and Lockhart- 0 Mummery, 1954; Brooke, 1954). A disposable $\frac{0}{\mathbb{D}}$ bag is made which dispenses with cleaning, though $\frac{\Omega}{\Phi}$ 
quite how to dispose of it the manufacturers have never explained-it is insoluble and may eventually cause obstruction to a normal sanitary system.

The patient seldom needs encouragement to take a full normal diet after leaving hospital, but a warning should be given to avoid eating the skins of certain fruits and vegetables, such as plums and tomatoes, since these can cause an obstructive bolus at the stoma. Extra salt should be taken whenever the small bowel discharges become more fluid. Full activity can be undertaken, for it is possible to play cricket, golf, tennis, football and even swim and dive without disturbing the bag, which being slender remains imperceptible even under a bathing dress. If any complication to the stoma arises which makes the management of the ileostomy impossible, the patient must return to hospital. Prolapse, retraction and fistula can only be treated by a revision of the ileost omy performed through a laparotomy incision; reconstructive operations confined to the stoma are ineffective and frequently lead to further trouble.

\section{BIBLIOGRAPHY}

BENJAMIN, D. (1954), Amer. F. Surg., 87, 27.

BROOKE, B. N. (1952), Lancet, ii, 102.

BROOKE, B. N. (1954), "Ulcerative Colitis and its Surgical Treatment,' Livingstone, Edinburgh.

COUNSELL, P. B., and LOCKHART-MUMMERY, H. E. (1954), Lancet, i, 113 .

GABRIEL, W. B. (1948), 'Principles and Practice of Rectal Surgery,' Lewis, London.

GABRIEL, W. B., and LLOYD-DAVIS, O. V. (1935), Brit. $\mathcal{F}$. Surg., 22, 520 .

HOFFMAN, E., and MACHT, A. (1954), Amer. F. Surg., 87, 140. LAHEY, F. H. (1951), Ann. Surg., 133, 726.

PATEY, D. W. (195I), Proc. roy. Soc. Med., 44, 423.

RANKIN, F. W. (1927), f. Amer. med. Ass., 89, 196 I.

\section{FLAT FOOT \\ By A. Graham Apley, F.R.C.S. \\ Consultant Orthopaedic Surgeon, Rowley Bristow Orthopaedic Hospital; Assistant to the Dept. of Orthopaedics, St. Thomas's Hospital, London.}

\section{Introduction}

Among the foot defects which cause disability, flat foot is one of the commonest. Normally the body weight is borne through two half-columns with the medial border of each foot raised from the ground (Wood Jones, 1943). The resulting arch may be high or low, yet still be healthy; but in flat foot the arch is not merely low, it has collapsed inwards (Fig. I).

\section{Aetiology}

\section{(a) Anatomical Causes}

There are four groups of anatomical peculiarities which predispose to flat foot. The inheritance of many of these peculiarities explains the frequent familial incidence of the disorder.

I. The lower limb may be wrongly' set' on the trunk. The entire limb may be externally rotated or the leg only may be rotated from the knee downwards In either case, the patient stands like Charlie Chaplin and the line of body weight falls too far medially (Fig. 2). As a result, when the body moves forward in walking the force of body weight imposes considerable strain upon the apex of the arch and tends to topple it over.

2. The leg may be wrongly 'set' on the thigh,
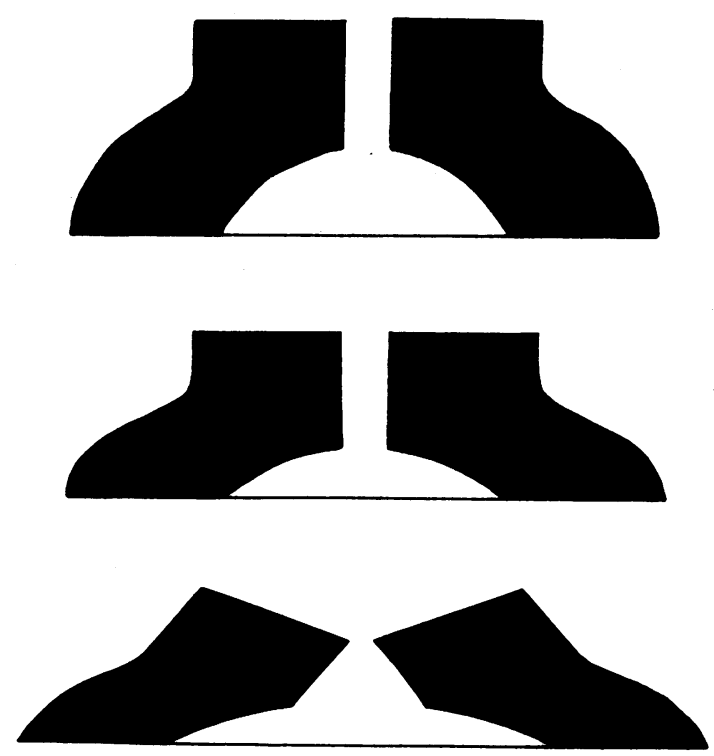

FIG. I

IST NORMAL ARCHES

2ND LOW ARCHES

3RD FLAT FEET 